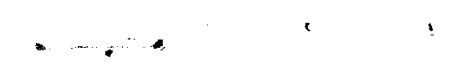

\section{DISTRIBUTED SENSING AND SHAPE CONTROL OF PIEZOELECTRIC BIMORPH MIRRORS}

\author{
James M. Redmond', Patrick S. Barney ${ }^{2}$, Tammy D. Henson ${ }^{3}$. \\ Sandia National Laboratories \\ Albuquerque, NM 87185 \\ ${ }^{1}$ Structural Dynamics and Vibration Control Department MS 0847 \\ ${ }^{2}$ Experimental Structural Dynamics Department MS 0557 \\ ${ }^{3}$ Optics and Emerging Technologies Department MS 0980
}

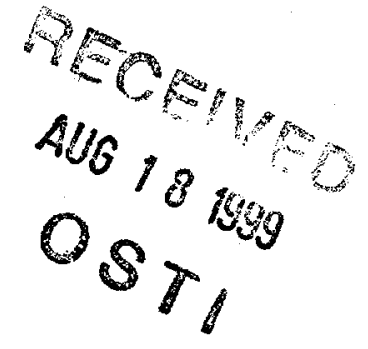

\author{
John A. Main and Jeffrey W. Martin \\ Department of Mechanical Engineering \\ University of Kentucky \\ Lexington, KY 40506-0108
}

\begin{abstract}
As part of a collaborative effort between Sandia National Laboratories and the University of Kentucky to develop a deployable mirror for remote sensing applications, research in shape sensing and control algorithms that leverage the distributed nature of electron gun excitation for piezoelectric bimorph mirrors is summarized. A coarse shape sensing technique is developed that uses reflected light rays from the sample surface to provide discrete slope measurements. Estimates of surface profiles are obtained with a cubic spline curve fitting algorithm. Experiments on a PZT bimorph illustrate appropriate deformation trends as a function of excitation voltage. A parallel effort to effect desired shape changes through electron gun excitation is also summarized. A one dimensional model-based algorithm is developed to correct profile errors in bimorph beams. A more useful two dimensional algorithm is also developed that relies on measured voltage-curvature sensitivities to provide corrective excitation profiles for the top and bottom surfaces of bimorph plates. The two algorithms are illustrated using finite element models of PZT bimorph structures subjected to arbitrary disturbances. Corrective excitation profiles that yield desired parabolic forms are computed, and are shown to provide the necessary corrective action.
\end{abstract}

\section{INTRODUCTION}

Recent military mishaps in the Balkans are indicative of a need for enhanced capability in remote sensing. In particular, the need arises for low cost surveillance satellites that can be quickly launched and positioned in orbit to monitor rapidly evolving events almost anywhere on the globe. However, the desire for low cost and flexibility is in opposition with the requirement for large apertures needed to ensure sufficient ground resolution and sensitivity. Such systems usually imply large. expensive launch vehicles to accommodate the size of the primary mirror. Development of a deployable mirror is one approach being considered to satisfy these conflicting requirements. Folded up and carried on a small booster, the mirtor would open to its full diameter in orbit. Unfortunately, the inherent flexibility of such a device makes it difficult to achieve optical quality surfaces, and this approach has therefore not yet been proven feasible.

Fueled by both NASA and DoD interests, much of the on-going research in deployable optics is focused on precision assembly of rigid mirror segments. While this approach shows promise for achieving near term sensing goals, it is unlikely that such a technique is capable of achieving long term areal density goals (on the order of $1 \mathrm{~kg} / \mathrm{m} 2$ ) needed to meet cost targets. Low areal densities can be achieved using inflatable optics (Marker, et al, 1998). However, this approach allows only limited shape correction through adjusting internal pressure, and suffers from the added complication of diffraction from passing light rays through a gaseous medium.

Structural shape control, which has been a topic of research among the Adaptive Structures community for two decades, offers many potential benefits to the growing field of large space-based optics. For example, some researchers have implemented smart material actuators to generate mirror distortions at discrete locations that correct for atmospheric disturbances (Hardy et al., 1997). Regional shape corrections are enabled by bonding active materials to the substrate, but still this technique provides shape control at only the actuator locations (Sato et al., 1980 and Steinhaus and Lipson, 1979). This limitation has led investigators to consider crafting the entire mirror body from electroactive materials. A bimorph mirror consisting of piezoelectric material layers with opposite poling will bend in response to an applied electric field and can therefore be deformed into desirable shapes (Morgan Matroc, 1993). Controlling specific regions on the mirror can be accommodated by segmenting the electrodes (Forbes et al., 1989), but the number of discrete electrodes needed to attain optical quality 


\section{DISCLAIMER}

This report was prepared as an account of work sponsored by an agency of the United States Government. Neither the United States Government nor any agency thereof, nor any of their employees, make any warranty, express or implied, or assumes any legal liability or responsibility for the accuracy, completeness, or usefulness of any information, apparatus, product, or process disclosed, or represents that its use would not infringe privately owned rights. Reference herein to any specific commercial product, process, or service by trade name, trademark, manufacturer, or otherwise does not necessarily constitute or imply its endorsement, recommendation, or favoring by the United States Government or any agency thereof. The views and opinions of authors expressed herein do not necessarily state or reflect those of the United States Government or any agency thereof. 


\section{DISCLAIMER}

Portions of this document may be illegible in electronic image products. Images are produced from the best available original document. 
from a depioyable mirror is prohibitive. An alternative excitation approach featuring an electron gun is being developed at the University Of Kentucky (Main, 1997 and Martin et al., 1998). With this technique, the charge at precise locations on the mirror is controlled by modulating beam intensity and the potential of a backside electrode.

As part of a collaborative effort between the University of Kentucky and Sandia National Laboratories, this approach is being explored as a method of obtaining optical quality surfaces from deployable mirrors. The four main research thrusts of this program are electron gun actuation, profile sensing, shape control algorithms, and space implementation issues. This paper summarizes some of the ongoing research in the areas of surface sensing and shape control. In particular, a technique for obtaining coarse resolution profiles of deformable mirrors is developed through modification of the Multibeam Optical Stress Sensing (MOSS) system (Floro et al., 1995 and Robinson, 1998). Originally developed to monitor stresses in semiconductor films during deposition processes, this technique can provide surface profiles by modifying the data reduction procedure. A shape estimation algorithm is developed and sample measurements from a cantilevered PZT bimorph subjected to various excitation levels are provided. For higher fidelity measurements needed to obtain optical quality surfaces, the Multi-wavelength heterodyne interferometry system being developed at Air Force Research Laboratories (McMakin et al, 1997 and Voelz et al., 1997) is being considered. This approach takes advantage of an optical beating phenomenon to synthesize long wavelength interferometry ideal for profiling highly distorted surfaces such as membrane mirrors.

Progress in developing shape control algorithms that take advantage of the distributed nature of the electron gun excitation is then summarized. One dimensional correction of bimorph beams is first developed using a model based approach. To accommodate structural and material uncertainty, a two dimensional approach is developed using measured voltage-curvature sensitivities. These algorithms are demonstrated using finite element models of PZT-5A bimorph structures subjected to arbitrary disturbances.

\section{SURFACE PROFILE MEASUREMENT}

\section{MOSS System Overview}

The Multi-beam Optical Stress Sensor (MOSS) system (Floro et al., 1995 and Robinson, 1998) was developed by researchers in the Nanostructure and Semiconductor Physics Department at Sandia for the purpose of monitoring stresses in thin films during their growth process. A schematic diagram of the MOSS system is shown in Figure 1. After some spatial filtering, a Helium-Neon laser beam is passed through an etalon. This optical device has a partially transmissive reflective coating on it's outer surfaces, allowing it to capture the light and reflect it internally. At each reflection, a portion of the beam leaks through the outer surface, resulting in an array of parallel beams that reflect off the sample. While only the one-dimensional implementation is presented here for simplicity, insertion of a second etalon askew to the first yields a two dimensional array of beams, enabling full surface profiling.

The reflected light rays are captured at the CCD camera positioned approximately $0.66 \mathrm{~m}$ above the sample surface. The essence of the MOSS system is computing the centroids of the spots on the camera pixel array and comparing the mean differential spacing of the spots to

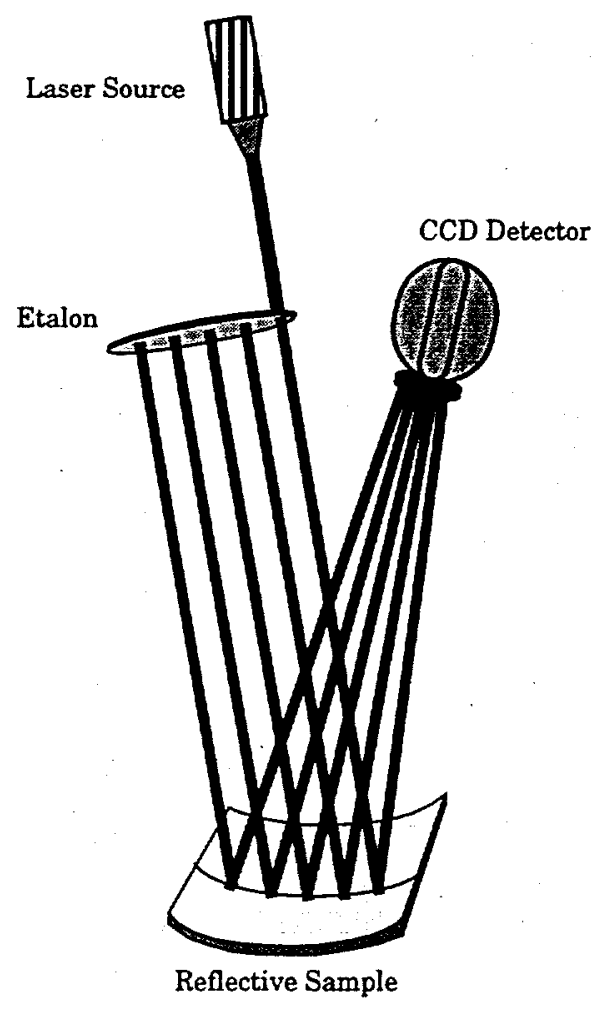

Figure 1. - Diagram of the Multiple beam Optical Stress Sensor (MOSS) system.

that of an optical flat. This measurement provides an estimate of the sample radius of curvature which is used to determine film stress. The sensitivity of the system is dependent on the geometric parameters of the hardware set sup (beam spacing, incidence angle, distance between sample and camera, etc.), but radii on the order of $2-5 \mathrm{~km}$ have been measured with the nominal test configuration.

Although not originally intended for this purpose, the data from the MOSS system can be reduced to yield local slope information on the sample to be used in estimating surface profile. This is done by recording the location of the reflected rays on the CCD camera and comparing them to the spot locations resulting from an optically flat reference sample. To illustrate, a single member of the laser array is shown in Figure 2 reflecting off a sample at location $x_{i}$. For a flat sample, the incident ray inclined at an angle $\alpha$ to the vertical will reflect off the surface along the dashed line, impinging on the detector plane at point $A$. If the sample is deformed with local slope $\theta_{i}$ as shown, then the reflected ray will deviate from the nominal path by an additional angle $2 \theta_{i}$, encountering the detector at location $\mathrm{B}$. The distance between the nominal and actual spot locations is given by

$$
\Delta_{i}=s_{i} \tan 2 \theta_{i}
$$

in which $s_{i}$ is the distance between the sample and the detector along the nominal ray path. The spot migration can be related to the surface slope by first recalling the surface slope definition

$$
\frac{d u\left(x_{i}\right)}{d x}=-\tan \theta_{i}
$$




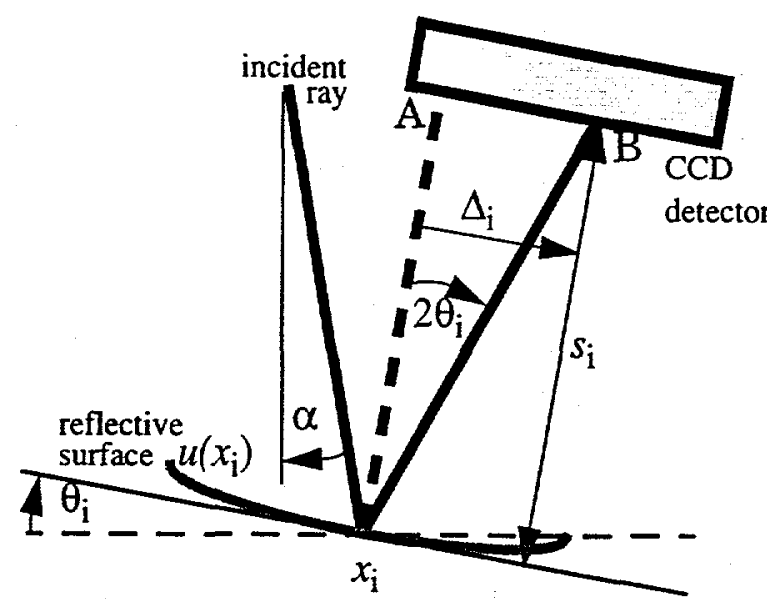

Figure 2. - Ray-tracing diagram for demonstrating discrete slope measurements on sample surface.

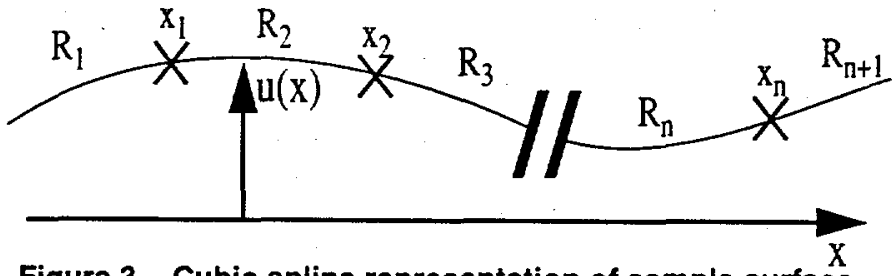

Figure 3. - Cubic spline representation of sample surface.

and using the double angle trigonometric identities to yield

$$
\frac{d u\left(x_{i}\right)}{d x}=\frac{s_{i}}{\Delta_{i}}-\left[\left(\frac{s_{i}}{\Delta_{i}}\right)^{2}+1\right]^{\frac{1}{2}}
$$

In this development the spot migration resulting from a pure translation of the surface has been neglected. This effect, while negligible for the cases considered in this study, may be a significant source of error for samples undergoing large deformations. For such cases, a second measurement of the reflected ray is needed to distinguish the contributions of the sample slope and displacement to the spot migration. Such a measurement can be readily obtained using a beam splitter and a second CCD camera.

\section{Surface Profile Estimation}

Once the slopes are known at $n$ discrete points on the sample surface, curve fitting algorithms can be used to estimate the distributed displacement profile. The approach taken here is to use cubic spline functions shown in Figure 3 to estimate the shape in the regions $R_{i}$ between the data points. These functions take the general form

$$
u(x)=a_{i} x^{3}+b_{i} x^{2}+c_{i} x+d_{i}, \quad i=1,2, \ldots n+1
$$

in which $u$ is the surface displacement and $a_{i} b_{i}, c_{i}, d_{i}$ are the initially undetermined spline coefficients representing $4(n+I)$ unknowns. A unique solution to the profile is given by enforcing the $3 n$ spline compatibility conditions (displacements, slopes, and curvatures

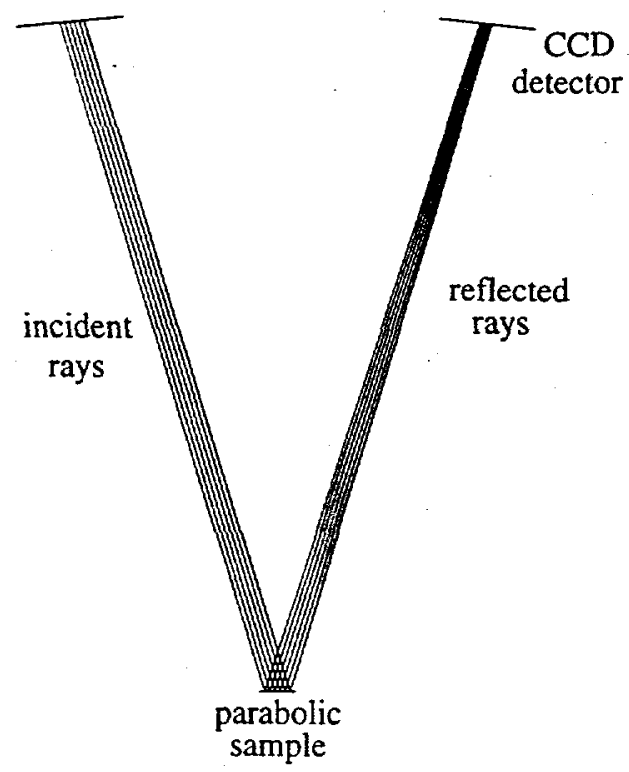

Figure 4. - Sample measurement with 6 rays and a parabolic sample clamped at the center

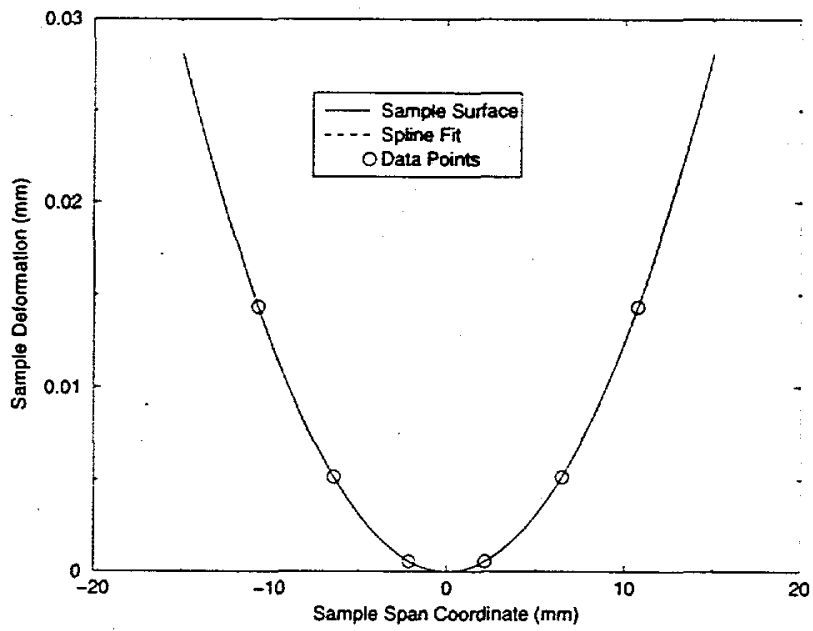

Figure 5. - Surface profile estimation using cubic spline routine with 6 discrete slope measurements.

equal at the spline junctions), incorporating the $n$ measured slopes, and imposing 4 boundary conditions at the sample end points.

To illustrate the curve fitting procedure, we first consider a $3 \mathrm{~cm}$ long sample clamped at the center and deformed in the shape of a perfect parabola with focal length of $2 \mathrm{~m}$. The sample is interrogated with 6 evenly spaced parallel arrays inclined at angle $\alpha=10^{\circ}$ relative to the vertical, as shown in Figure 4. The rays reflecting off the concave surface converge as they approach the CCD camera located $1 \mathrm{~m}$ from the sample surface. The spot locations are recorded and compared to those of an optically flat sample. The resulting $\Delta_{i} \mathrm{~s}$ are used to estimate slope (according to equation 3 ) which are then used to produce the surface profile shown in Figure 5. In the absence of surface errors and measurement noise, the six data points provide an exact fit to the surface. However, much more data may be needed to adequately 


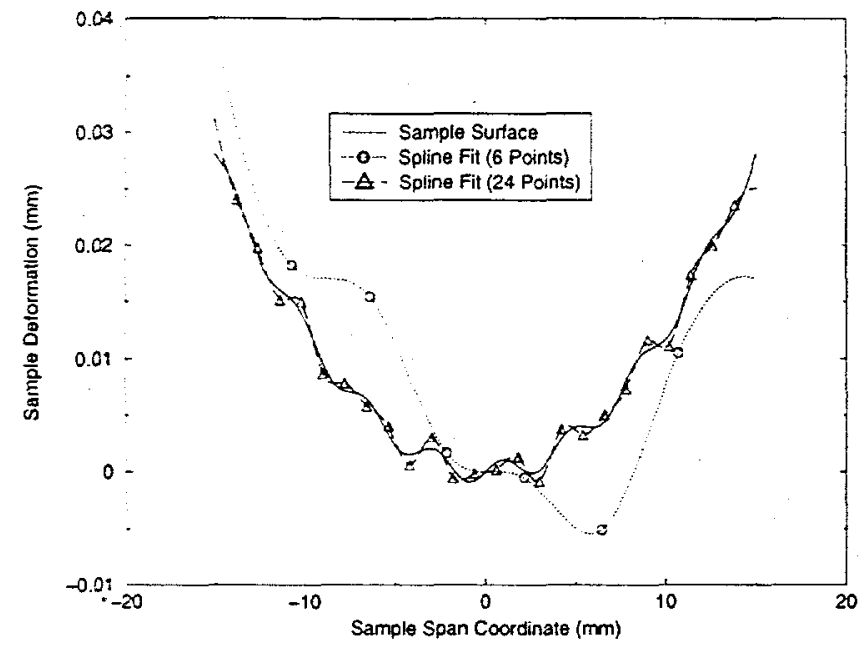

Figure 6. - Profile estimation of distorted sample surface using 6 and 24 discrete data measurements.

represent the profile of highly distorted surfaces. For example, consider a sinusoidal distortion superimposed on the parabolic proffle of the previous example. The error curve with period $3.75 \mathrm{~mm}$ and amplitude of $1 \mu \mathrm{m}$ produces small changes in the surface slope at the measurement locations. As shown in Figure 6, considerable errors are present in the profile estimated using only 6 discrete slope measurements. The error, however. is significantly reduced by the inclusion of additional data points. With 24 slope measurements, the general profile of the distorted surface is captured as indicated in the figure.

Although the MOSS systems default configuration allows for only four discrete slope measurements, increased resolution can be obtained by altering the etalon to reduce the beam spacing, and stepping the sample underneath the beam array. This approach was taken in the following experimental investigation.

\section{Experimental Demonstration of MOSS Based Profiler}

To illustrate the utility of the proposed surface estimation procedure on a real sample, a series of experiments was conducted on a deformable piezoelectric actuator subjected to varying excitation levels. The PZT-5A bimorph actuator used in the experiment was manufactured by Morgan Matroc (model \#61620). Featuring parallel wiring and full nickel electrodes, the actuator had nominal dimensions of $5 \times 2 \times 0.06 \mathrm{~cm}$. One side of the actuator was polished to obtain a sufficient level of reflectivity to enable interrogation by the MOSS system. In all of the experiments conducted, the actuator was clamped at one end, exposing approximately $3 \mathrm{~cm}$ in a cantilevered configuration. To simplify the connection of electrodes to the sample, only the upper plate of the bimorph was excited. With the bottom half of the actuator acting as a constraining layer, applying a potential across the upper plate caused a bending deformation that altered the path of the reflected beams. An array of four parallel beams with horizontal spacing of $1.4 \mathrm{~mm}$ and an incident angle of $\alpha=2.5^{\circ}$ were tracked by the CCD camera located $0.66 \mathrm{~m}$ above the surface of the sample. At each excitation level. the sample was stepped under the beam array to provide additional data points. Depending on the degree of sample deformation, varying amounts of data were obtained before the reflected arrays passed out of range of the stationary camera.

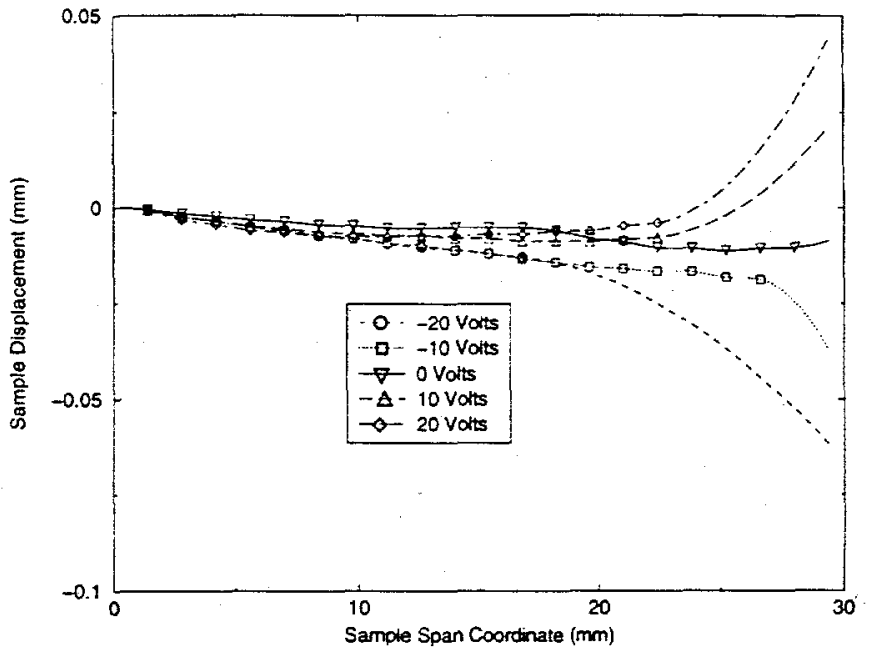

Figure 7. - Profile estimation of sample surface at varying excitation levels.

As shown in Figure 7, interrogation of the zero volt sample yielded twenty data points which were then used in the cubic spline surface profiler. Some initial distortion was present in the sample as a consequence of residual stresses induced in the fabrication and polishing procedures. Increasing the excitation level (positively) resulted in an upward curvature. Tip deflections of 30 and $50 \mu \mathrm{m}$ above the zero volt reference were estimated for the 10 and 20 volt cases, respectively. These estimates, however, were obtained using only 16 data points in the curve fitting procedure, since beam reflections on the outboard portion of the sample fell out of range of the CCD camera. Therefore, the profile in this region is an extrapolation from the last slope measurement available at $x=22.5 \mathrm{~mm}$.

Negative excitations produced a downward curvature in the sample as anticipated. At -10 volts, 19 data points were used to obtain a relative tip deflection of $-0.03 \mathrm{~mm}$. The deflection estimate increased to -0.06 $\mathrm{mm}$ at -20 volts, though only 12 data points were available

\section{Summary of MOSS Results}

While the existing MOSS system was suitable for demonstration purposes, several modifications are needed to realize the full potential of this approach for measuring sample deflections. In the experiment conducted, moderate excitation levels produced surface distortions sufficient to steer the beams out of the range of the detector. Therefore, a method for expanding the range of the slope measurement is needed to maximize the surface discretization. Moving the camera closer to the sample would expand it's range, but at the expense of resolution. Another approach that yields the same result is to employ additional optical elements that steer the beam back onto the camera, but expanding the dynamic range requires a CCD camera with a larger sensing array. For even larger deformations, the camera could be mounted on a linear stage, enabling tracking of the reflected beams.

A second issue requiring additional consideration is that of slope errors resulting from surface displacements. As mentioned previously, migration of the reflected beams across the surface of the detector are attributed solely to changes in the surface slope. While this assumption is valid for small surface distortions, surface translations can produce 
beam migrations that are indistinguishable from migrations resulting from steering the beams via slope changes.

\section{SHAPE CONTROL}

Converting measured profiles to corrective electron gun action is the focus of the control algorithm development effort. Electron gun excitation enables the high resolution surface curvature corrections. Thus, in the absence of uncertainties in the material parameters and dimensions, an exact corrective profile can be computed using piezoelectric bimorph models. For the more general case, test derived distributed voltage-curvature relationships can be integrated into the correction algorithm.

\section{One Dimensional Shape Correction}

Neglecting the effects of glue layers and electrodes, the internal moment resulting from excitation of a uniform PZT bimorph beam is given by (Martin et al., 1998)

$$
M(x)=\int_{-t / 2}^{0} e_{31} E_{3}(x) b z d z-\int_{0}^{t / 2} e_{31} E_{3}(x) b z d z
$$

in which $b$ and $t$ are the beam width and thickness, $z$ is the distance form the midpoint, $e_{31}$ is the piezoelectric constant, and $E_{3}(x)$ is the axially distributed electric field. Assuming parallel wiring (ground plane at center) and separating the elastic and piezoelectric effects, the moment reduces to

$$
M(x)=E d_{31} b \frac{t}{2} V(x)
$$

in which $E$ represents Young's modulus, $d_{31}$ is the more familiar piezoelectric constant, and $V(x)$ is the voltage applied across the top surface and the center ground plane.

The corrective action of the PZT is superimposed on the deformation of the structure resulting from mechanical disturbances. For a uniform beam, the moment yields a corrective curvature according to

$$
u "(x)=\frac{M(x)}{E I}
$$

where I is the area moment of inertia of the PZT cross section. Recognizing that the desired curvature correction is the difference between the desired curvature $u_{d}{ }^{\prime \prime}(x)$ and the measured curvature $u_{m}{ }^{\prime \prime}(x)$. equating Equations 6 and 7 yields

$$
V_{c}(x)=\frac{t^{2}}{6 d_{31}}\left(u_{d}{ }^{\prime \prime}(x)-u_{m}{ }^{\prime \prime}(x)\right)
$$

in which $V_{c}(x)$ is the distributed corrective excitation profile.

\section{Two Dimensional Shape Correction}

Practical optical applications such as remote sensing require independent control of the surface curvature in two directions in order to achieve complicated profiles such as a paraboloid from a general initial profile. This is difficult to realize in practice since both $x$ and $y$

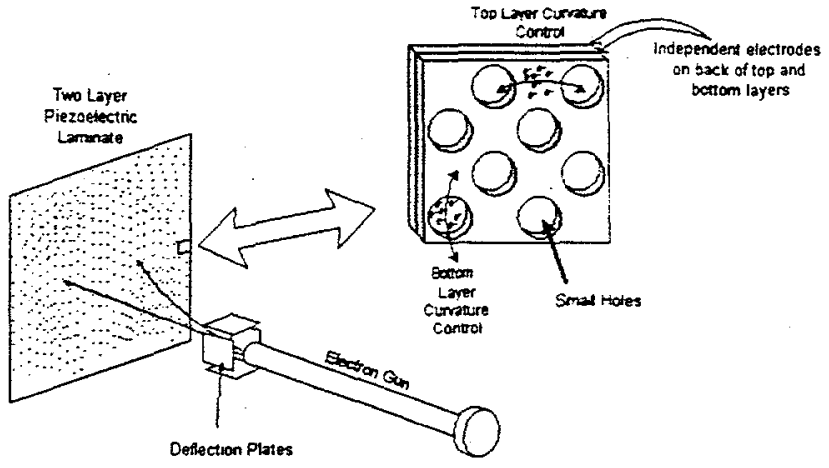

Figure 8. - Conceptual design for a bimorph mirror amenable to independent layer control using a single electron gun excitation source.

curvatures are generally induced by a single excitation across the thickness of the piezoelectric material. One possible solution which requires independent excitation of the top and bottom layers of a bimorph structure is now presented.

The relationship between curvatures and the excitations at a point on a bimorph structure involve a complex coupling of the electromechanical properties of the piezostructure. In simple terms, this relationship can be expressed as

$$
\left[\begin{array}{l}
\gamma_{x}(x, y) \\
\gamma_{y}(x, y)
\end{array}\right]=\left[\begin{array}{ll}
F_{t}(x, y) & F_{b}(x, y) \\
G_{t}(x, y) & G_{b}(x, y)
\end{array}\right]\left[\begin{array}{l}
V_{t}(x, y) \\
V_{b}(x, y)
\end{array}\right]
$$

in which $\gamma_{x}$ and $\gamma_{y}$ are the curvatures along the $\mathrm{x}$ and $\mathrm{y}$ directions, $V$, and $V_{b}^{x}$ are the top and bottom surface excitations, $F$ and $G$ are the coupling coefficients. Although the coupling coefficients naturally vary over the surface due to slight variability in the structural and material properties, they are nominally the same for materiais displaying uniform piezoelectric properties ( $d_{31}=d_{32}$ ). Therefore, excitation of the top or bottom surface will produce similar curvature changes in both the $x$ and $y$ directions. In this case, the coefficient matrix of Eq. 9 is singular, and it is not possible to determine a corrective excitation profile to negate inherent curvature errors.

To circumvent this difficulty, we allow the bimorph to consist of a preferentially biased material such that $d_{31} \neq d_{32}$, an effect that can be achieved by straining the material during poling. Then, offsetting the layers of the bimorph by 90 degrees renders the coefficient matrix of equation 9 nonsingular. Top an bottom surface excitation profiles can then be uniquely determined for random curvature errors. One approach to achieving independent excitation of the top and bottom layers of the bimorph using a single electron gun is illustrated conceptually in Figure 8. Small holes are included in the top layer to provide access to the bottom layer. The impact of the perforated surface on the optical performance of the mirror is under further investigation.

For a uniform structure, the coefficient matrix of Equation 9 can be determined analytically, but an experimental calibration procedure is favored to accommodate uncertainties in material and fabrication parameters. This procedure is illustrated in the second of the following examples. 


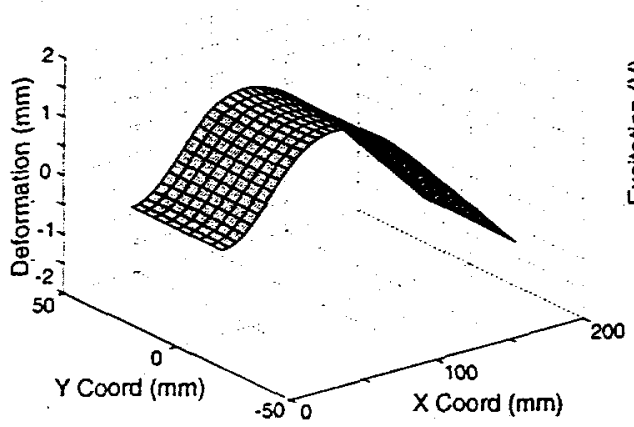

a. initial deformed shape

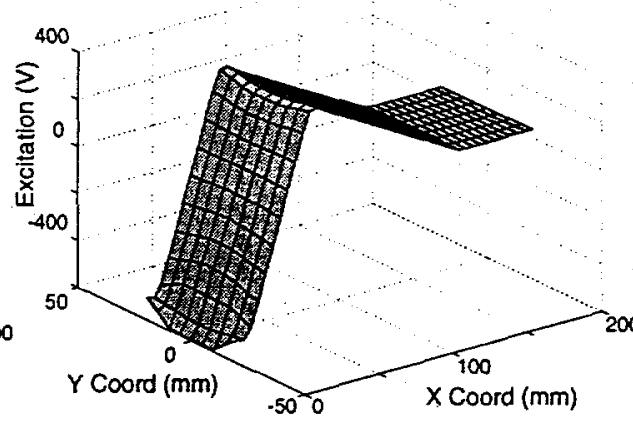

b. corrective excitation profile

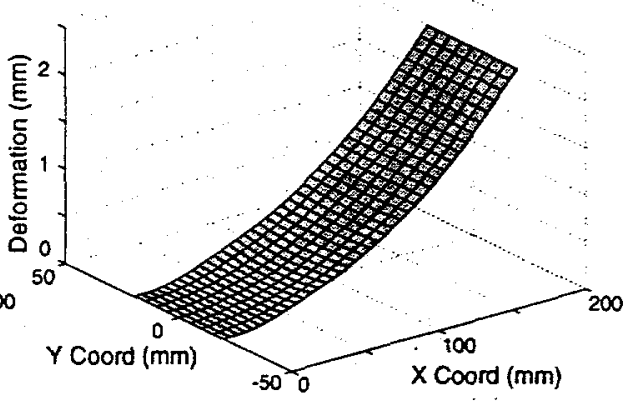

c. final parabolic shape

Figure 9. - One dimensional shape control illustration using distributed excitation source on a cantilevered PZT bimorph beam.

Table 1. - Sample parameters for one-dimensional cantilevered bimorph example.

\begin{tabular}{|c|c|}
\hline Material & PZT 5A \\
\hline Dimension & $20 \times 4 \times 0.06 \mathrm{~cm}$ \\
\hline$d_{31}$ & $-171 \times 10-12 \mathrm{~m} / \mathrm{V}$ \\
\hline$d_{32}$ & 0 \\
\hline Young's Modulus & $6.1 \times 10^{10} \mathrm{~N} / \mathrm{m}^{2}$ \\
\hline wiring & parallel \\
\hline Desired Curvature & $0.001 \mathrm{~cm}^{-1}$ \\
\hline
\end{tabular}

\section{Shape Correction lllustrations}

Experimental demonstration of the shape correction algorithms requires a vacuum chamber to accommodate the electron gun excitation. Without such a test facility, individual electrodes and lead wires must be attached to the surface of the bimorph to provide at best a coarse corrective capacity. An electron gun test bed featuring an 18" diameter vacuum chamber is under development at Sandia and should be completed by late Summer of 1999. However, the shape control algorithms can be presently illustrated using a piezoelectric finite element model coupled to external control algorithms.

Cantilevered Bimorph. We first consider a cantilevered PZT5A bimorph beam whose parameters are summarized in Table 1 (Morgan Matroc, 1993). A layered shell model developed in Abaqus (HKS. 1998) was subjected to initial disturbances yielding the error shape shown in Figure 9a. For this example, a desired constant curvature of $0.001 \mathrm{~cm}^{-1}$ along the beam span is assumed. The element strains on the upper surface were interpolated to nodal strains and converted to curvature measurements according to

$$
u_{m}^{\prime \prime}(x)=\frac{\varepsilon_{11}^{t o p}(x)-\varepsilon_{11}^{b o t}(x)}{t}
$$

Table 2. - Sample parameters for two-dimensional bimorph plate example.

\begin{tabular}{|c|c|}
\hline Material & PZT $5 \mathrm{~A}$ \\
\hline Dimension & $10 \times 10 \times 0.06 \mathrm{~cm}$ \\
\hline$d_{31}$ & $-171 \times 10^{-12} \mathrm{~m} / \mathrm{N}$ \\
\hline$d_{32}$ & $-57 \times 10^{-12} \mathrm{~N} / \mathrm{m}^{2}$ \\
\hline Youngs Modulus & $6.1 \times 10^{10} \mathrm{~N} / \mathrm{m}^{2}$ \\
\hline wiring & parallel \\
\hline Desired Curvature & $0.004 \mathrm{~cm}^{-1}$ \\
\hline
\end{tabular}

in which $t$ is the bimorph thickness $\varepsilon_{11}^{t o p}(x)$ and $\varepsilon_{11}^{b o t}(x)$ are the axial strains on the top and bottom surfaces.

From equation 8 , the corrective excitation profile for the top surface was determined as shown in Figure $9 \mathrm{~b}$. For this one dimensional example, it is sufficient to show the single profile with the implicit assumption that a similar profile of opposite polarity is applied to the bottom surface. Then, a second finite element analysis exercising the electromechanical coupling of the piezoelectric with the corrective excitation profile was completed, yielding the corrected profile shown in 9c.

Although the control algorithm illustrated in this example provides useful insight into the shape correction methodology, it is ill suited to the general problem of mirror shape control. The algorithm cannot correct for an arbitrary disturbance due to its one dimensional structure, and its reliance on an analytical model for determining corrective voltage makes it difficult to achieve optical quality surfaces in the presence of material and structural uncertainty.

Bimorph Plate. Circumventing the short comings of the one dimensional algorithm, we now illustrate the two dimensional algorithm using a calibration procedure for determining the appropriate coupling coefficients. A finite element model of a square bimorph plate with parameters summarized in Table 2 was fixed at its center point and subjected to an arbitrary loading. The resulting deformed profile is shown in Figure 10a, revealing a curvature error representative of the 


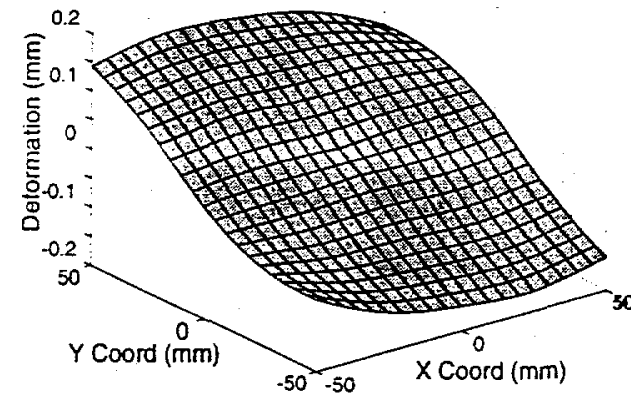

a. initial deformed shape

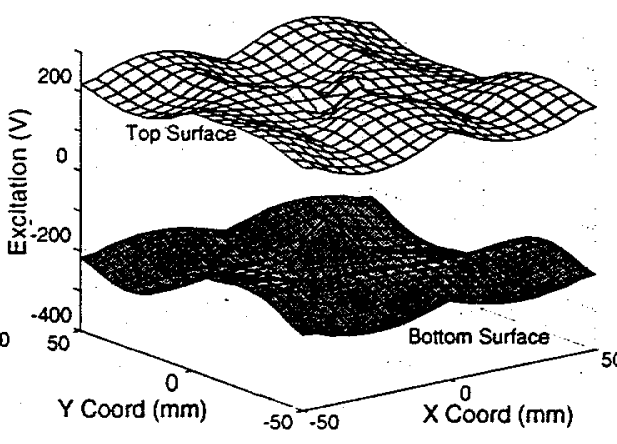

b. corrective excitation profiles

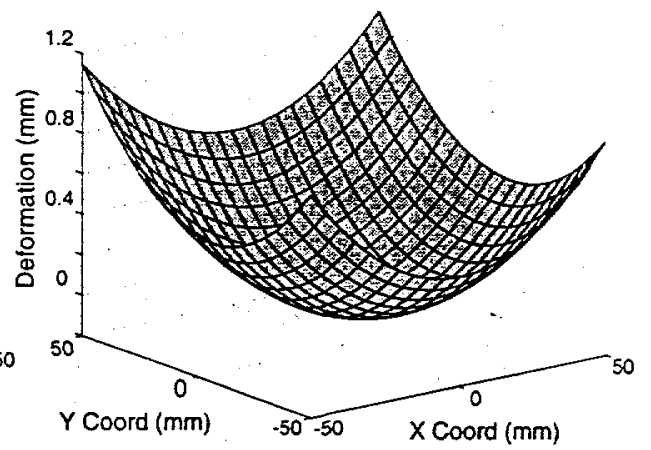

c. final paraboloidal shape

Figure 10. - Two Dimensional distributed shape control illustration using a distributed excitation source on a square PZT bimorph plate featuring preferential layer deformations and independent layer control.

general mirror problem. The distributed coupling coefficient functions of Equation 9 were determined by applying uniform voltages to the top and bottom surfaces of the deformed structure and extracting the curvature sensitivities. This procedure mimics the calibration step to be implemented in future experiments. The corrective voltages needed to obtain the desired curvature of $0.004 \mathrm{~cm}^{-1}$ at each node point were calculated by multiplying the curvature error present in the initial deformed structure by the inverse of the local coefficient matrix. The resulting top and bottom surface excitations are shown in Figure 10b, revealing significant variation in the profiles, particularly in the vicinity of the fixed center point. A final finite element solution exercising the piezoelectric properties of the model was computed, yielding the corrected profile shown in Figure 10c. Only minor discrepancies in the corrected profile are evident and can be attributed to the relatively coarse discretization of the surface in comparison to actual electron gun spot size.

\section{CONCLUSIONS}

This paper summarized sensing and shape control research toward the realization of deformable piezoelectric bimorph mirrors using electron gun excitation. First, a coarse profiling system that uses discrete measurements of sample slope obtained by reflecting light off its surface was summarized. A cubic spline curve fitting algorithm was invoked to yield profile estimations. Experiments on a cantilevered PZT bimorph mirror yielded profiles consistent with excitation patterns. This technique can be easily interfaced with a vacuum chamber, and can therefore be a useful tool for developing electron gun excitation strategies.

On-going research into the development of viable algorithms for correcting surface profiles in bimorph mirrors was then described. Onedimensional model based correction was demonstrated on a finite element model of a cantilevered beam. More useful to the task of mirror shape control, a two-dimensional algorithm using test derived curvature sensitivities was presented. Essential to this effor is the development of a bimorph whose layers exhibit preferential deformation. Coupled with independent excitation of the two layers, this provides the necessary control authority to correct arbitrary two-dimensional deformations. The algorithm was implemented on a finite element model of a square plate subjected to an arbitrary disturbance. After a completing a calibration procedure, distributed excitation profiles for the top and bottom surfaces of the mirror were computed and shown to achieve the desired profile.

Validation experiments are currently being planned for an electron gun/vacuum chamber testbed being developed at Sandia in collaboration with the University of Kentucky. Realization of general mirror shape correction requires additional research in converting top and bottom surface excitation profiles into electron gun control parameters (power, dwell time).

\section{ACKNOWLEDGEMENTS}

The authors wish to thank John Hunter of Sandia for operating the MOSS system and providing the resulting data in the needed format. Sandia National Laboratories is a multiprogram laboratory operated by Sandia Corporation, a Lockheed Martin Company, for the United States Department of Energy under Contract DE-ACO4-94AL85000.

\section{REFERENCES}

Floro, J.A., Chason, E., and Lee, S.R., 1995, "Real Time Measurement of Epilayer Strain Using a Simplified Wafer Curvature Technique," SAND95-1731C, Sandia National .Laboratories, Albuquerque, NM.

Forbes, F., Roddier, F., Poczulp, G., Pinches, C., Sweeny, G., and Dueck, R., 1989, "Segmented Bimorph Deformable Mirror," Journal of Physics E: Scientific Instruments, Vol. 22, pp. 402-405.

Hardy, J.W., Lefebyre, J.E., and Koliopoulos, 1977, "Real-Time Atmospheric Compensation," Joumal of the Optical Society of America, Vol. 67, No. 3, pp. 360-369.

Hibbitt, Karlsson, \& Sorensen, Inc, 1998, Abaqus Standard/User's Manual, version 5.8 .

Main, J.A., "Control of Distributed Actuators Without Electrodes." Proceedings of the 1997 ASME International Mechanical Engineering Congress and Exposition, Dallas, TX. November 14-19, 1997.

Marker, D.K., Carreras, R.A., Wilkes, J.M., Jenkins. C.H., Duneman, D.C., Rotge, J. R., and Hogge, C.B., 1998. “Optical 
Evaluation of Membrane Mirrors with Curvature," Proceedings of SPIE, Vol. 3430 , p. 202-208.

Martin, J.W., Main, J.A., and Nelson, G.C., "Shape Control of Deployable Membrane Mirrors," Proceedings of the 1998 ASME International Mechanical Engineering Congress and Exposition, Anaheim, CA, November 15-20, 1998.

McMakin, L., Voelz, D.G., and Fetrow, M.P., 1997, "Multiple Wavelength Heterodyne Array Interferometry," Optics Express, Vol. 1. No. 11, pp. 332-337.

Morgan Matroc, Inc, 1993, "Guide to Modern Piezoelectric Ceramics".

Robinson, K., "Optical Sensor Monitors Film Growth," Photonics Technology World, September 1998, pp. 51-52.

Sato, T., Ishida, H., and Ikeda, O., 1980, "Adaptive PVDF Piezoelectric Deformable Mirror System," Applied Optics, Vol. 19, No. 9, pp. 1430-1434.

Steinhaus, E., and Lipson, S.G., 1979, "Bimorph Piezoelectric Flexible Mirror," Journal of the Optical Society of America, Vol. 69, No. 3, pp. 478-481.

Voelz, D.G., McMakin, L., Boger, J.K., and Fetrow, M.P., 1997, "Double Exposure Heterodyne Imaging for Observing Line-of-Sight Deformation," Optics Letters, Vol. 22, No. 13, pp. 1027-1029. 